\title{
DIETETIC THERAPY AIMED TO RESTART MENSTRUAL PERIOD IN PATIENTS AFFECTED BY ANOREXIA NERVOSA IN WEIGHT RECOVERY
}

Policola C. ' ', Musca T. ${ }^{2}$, Sicignano L.L. ${ }^{3}$, Cefalo C.M.A. ${ }^{\text {, }}$ Meniconi P. ${ }^{2}$, Lassandro

\section{Background}

Anorexia Nervosa (AN) is a severe eating disorder characterized by significantly low body weight and fear of gaining weight. It affects about 1 in 250 females and 1 in 2000 males and usually starts in adolescence or young adult life even if it can occur in childhood and later life. Amenorrhea is a very frequent feature in AN and it could persist after normalization of body mass index (BMI). A well represented fat mass and an adequate body composition (BC) are necessary for resumption of menstrual periods. Patients affected by AN, although in the remission phase, fear of becoming fat and refuse to gain weight.

\section{Aim}

In our study we evaluated the effect of a normo-caloric $30 \%$ fat diet on BC in patients affected by AN in weight recovery and its effects in resuming menstrual cycles.

\section{Material and Methods}

We studied 12 patients (tab. 1): 6 patients (group 1) with $A N$ and hypothalamic amenorrhea matched with 6 (group 2) healthy controls, homogeneous for age, body weight and BMI. The following laboratory tests were performed: FSH and LH basal and after GnRH stimulation, Oral Glucose Tolerance Test, fT3, fT4, TSH, basal cortisol, 17beta-estradiol, 17OH progesterone, testosterone, DHEAS, SHBG, Vitamin D and PTH. All subjects underwent BIA and DEXA. Group 1 had been on 30\% lipids, 22\% proteins and $48 \%$ carbohydrates diet-regime for 6 months.

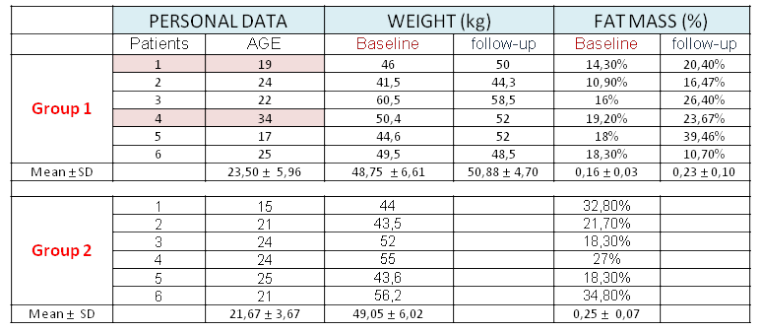

patients with spontaneous menstruations restart

Tab. 1: Characteristic of study population

\section{Results}

At baseline the content of adipose tissue in the group 1 was significantly underrepresentated $(p=0.02)$; after 6 months of diet therapy, the fat mass level was increased $(p=0.03)$. Furthermore GnRH stimulated FSH levels decreased and LH levels significantly increased $(p=0.05)$ compared to the baseline. Insulin levels after Oral Glucose Tolerance Test were significantly decreased at time 90' and 120' ( $p=0.01 ; p=0.049)$ compared to the baseline. In two of six amenorrhoeic patients spontaneous menstruations restarted.

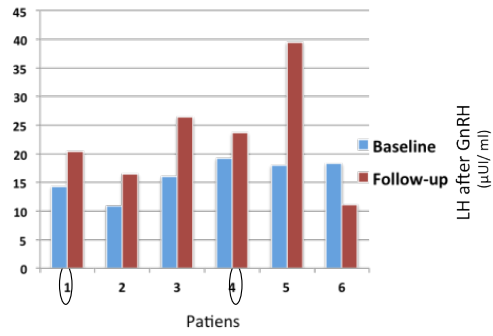

Fig. 1: \%fat in group 1 at baseline and follow-up

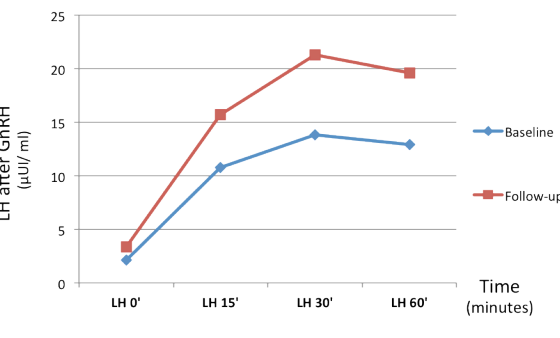

Fig. 2: LH after GnRH in group 1 at baseline and follow-up

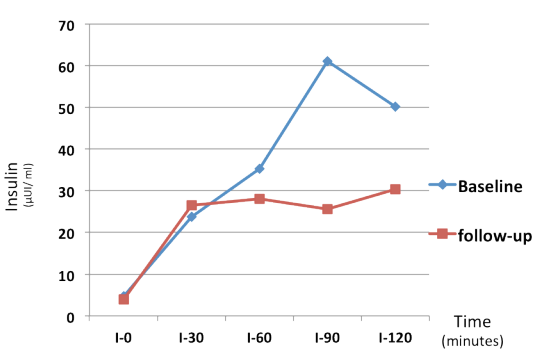

Fig. 3: Insulinemia after OGT in group 1 at baseline and follow-up

\section{Conclusion}

The increase of fat mass obtained after 6 months 30\% fat diet regime improved hormonal levels, reduced insulin resistance in all the patients and allowed the resuming of menses in 2 of 6 amenorrheic patients without a significant weight increase. It should be essential to study the effects of a targeted dietotherapy in the long term and increase our study-population. 